\title{
Hyperbolic Cosine Latent Trait Models for Unfolding Direct Responses and Pairwise Preferences
}

\author{
David Andrich
}

Murdoch University

\begin{abstract}
The hyperbolic cosine unfolding model for direct responses of persons to individual stimuli is elaborated in three ways. First, the parameter of the stimulus, which reflects a region within which people located there are more likely to respond positively than negatively, is shown to be a property of the data and not arbitrary as first supposed. Second, the model is used to construct a related model for pairwise preferences. This model, for which joint maximum likelihood estimates are derived, satisfies strong sto-
\end{abstract}

chastic transitivity. Third, the role of substantive theory in evaluating the fit between the data and the models, in which unique solutions for the estimates are not guaranteed, is explored by analyzing responses of one group of persons to a single set of stimuli obtained both as direct responses and pairwise preferences. Index terms: direct responses, hyberbolic cosine model, item response theory, latent trait models, pair comparisons, pairwise preferences, unfolding models.

The cumulative process and the unfolding process are the two main response processes for dichotomous responses in the measurement of social-psychological variables. In the cumulative response process, the probability of a positive response is a monotonic function of the relevant parameters; in the unfolding response process, it is a single-peaked function. For each of these two processes, there are two basic data collection designs: the direct-response design and the pair-comparison design. In the direct-response design, persons respond directly to items; in the pair-comparison design, they compare items in pairs. This paper is concerned with two related models for the unfolding response process-one for the direct-response design and one for the pair-comparison design. For both designs, the responses are dichotomous and the response model is an item response theory (IRT) or latent trait theory model. Accordingly, both the person and the item are hypothesized to be located on the same latent continuum. Although unfolding models can be extended to characterize more than one dimension, this paper is concerned only with unidimensional models.

The unfolding model for direct responses presented here is the hyperbolic cosine model for direct responses (HCMDR) introduced by Andrich \& Luo (1993). The model has two distinctive features. First, the symmetry of the single-peaked response function $(\mathrm{RF})$ is captured naturally by the hyperbolic cosine function. Second, it includes two parameters for each item. The first is the usual location parameter; the second characterizes the range around the location in which a person is most likely to give a positive response.

This paper briefly reviews this rationale for the HCMDR, and then extends it in three directions. First, Andrich \& Luo's (1993) specialization of the second parameter to an arbitrary value is reconsidered. It is shown that it is not arbitrary but is a property of the data. Second, the HCMDR is used to construct an elegant model for pairwise preferences in which the symmetry of the single-peaked $\mathrm{RF}$ is again characterized by the hyperbolic cosine function. Third, the peculiar problems that arise with models for unfolding because 
two person locations give the same probability of a positive (and negative) response are examined, and the role of substantive theory in helping to overcome these problems in testing the fit between the models and data is articulated. This articulation is exemplified by comparing parameter estimates using the two models from responses of the same group of persons to the same set of items according to both the direct-response design and the pair-comparison design.

\section{The Unfolding Response Process and Direct Responses}

Figure 1 shows both the single-peaked RF for a positive response of a person to an item and the complementary RF for a negative response (the solid lines), as well as two other functions. Table 1 shows a deterministic unfolding pattern for five items ordered according to their affective values.

Figure 1

Resolution of the Disagree Response Into its Constituent Components-DB and DA

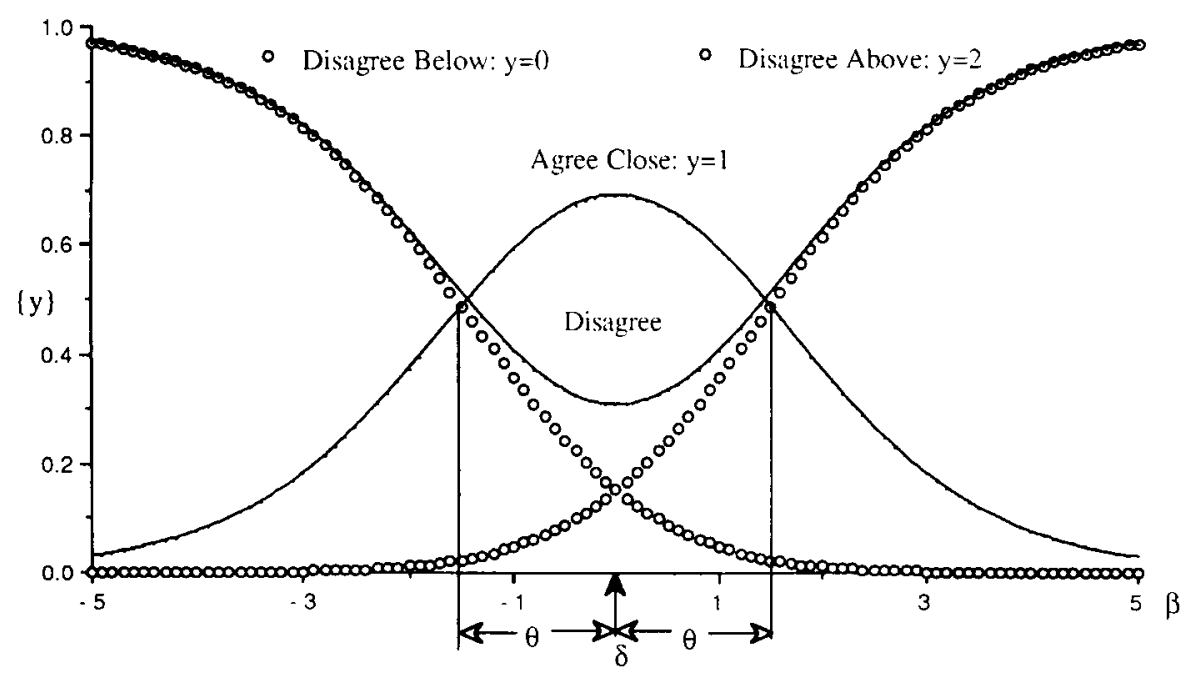

Although Thurstone's $(1927,1928)$ law of comparative judgment (LCJ) for the pair-comparison design and the cumulative RF introduced the idea of the unfolding response process for the measurement of attitude following a scaling of statements, the study of unfolding is actually associated with Coombs (1964).

Table 1

A Deterministic Unfolding Response Pattern for Five Items

\begin{tabular}{ccccc}
\hline \hline \multicolumn{5}{c}{ Item } \\
\hline 1 & 2 & 3 & 4 & 5 \\
\hline 0 & 0 & 0 & 0 & 0 \\
1 & 0 & 0 & 0 & 0 \\
1 & 1 & 0 & 0 & 0 \\
0 & 1 & 1 & 0 & 0 \\
0 & 0 & 1 & 1 & 0 \\
0 & 0 & 0 & 1 & 1 \\
0 & 0 & 0 & 0 & 1 \\
0 & 0 & 0 & 0 & 0 \\
\hline
\end{tabular}


However, Coombs' comprehensive studies of the unfolding response process for both the direct-response and the pair-comparison designs were carried out within a deterministic framework, much like Guttman's (1950) studies of the cumulative response process. Unfortunately, even with a deterministic structure, when more than four items are involved the data analysis becomes extremely complex for the unfolding response process. Explicit probabilistic and statistical models involving single-peaked functions for direct responses (e.g., Andrich, 1988; Davison, 1977; Post, 1992; van Schuur, 1984, 1989) and for pair-comparisons (e.g., Carroll, 1972; Cooper \& Nakanishi, 1983; DeSarbo \& Hoffman, 1986; Poole, 1984; Schönemann, 1970; Schönemann \& Wang, 1972; Sixtl, 1973) have been introduced. Bossuyt (1990) provided a comprehensive summary of probabilistic unfolding models for pair-comparisons.

The measurement of attitude (Thurstone's original application of the unfolding response process) was studied here using one of Thurstone's scales. However, the unfolding process also has been applied routinely in studying development along a continuum; for example, in psychological development (Coombs \& Smith, 1973); development in learning goals (Volet \& Chalmers, 1992); social development (Leik \& Matthews, 1968); in general preference studies (Coombs \& Avrunin, 1977); and in political science where preference and choice among alternatives is an essential process (van Blokland-Vogelesang, 1991; van Schuur, 1987).

\section{The HCMDR}

Consider a dichotomous response of Agree or Disagree of a person to the statement $I$ think capital punishment is necessary but I wish it were not. This statement reflects an ambivalent attitude toward capital punishment. If the person's location, termed the ideal point by Coombs (1964), is close to that of the statement, then the person will tend to Agree to the statement; if a person's ideal point is far from that of the statement-either very much for or very much against capital punishment-then the probability of the person selecting the Agree response will decrease and that of selecting the Disagree response will increase correspondingly.

Let $\beta_{n}$ be the location of person $n$ and $\delta_{i}$ be the location of statement $i$. Then formally, if $\beta_{n}<<\delta_{i}$ or $\beta_{n} \gg>$ $\delta_{1}$, the probability of a Disagree response tends to 1.0. Thus, the Disagree response occurs for two latent reasons-either the person has a much stronger attitude against capital punishment or the person has a much stronger attitude for capital punishment than that reflected by the statement. The RFs in Figure 1 plotted as "circle lines" show the resolution of the single Disagree response into these two constituent components.

Although the Disagree response gives no indication of the direction in which the person appears to be distant from the statement, the task is to estimate the relative locations of the statement and the person on the continuum, and this requires reconstructing the direction from the Disagree response. It was to describe this task that Coombs (1964) introduced the term unfolding. That is, the two directions that are effectively folded onto each other in a Disagree response need to be unfolded.

The resolution of the Disagree response into its two components shows that there are three latent responses that correspond to the two manifest responses of Agree or Disagree: (1) Disagree because the person considers himself or herself below the location of the statement [Disagree below (DB)], (2) Agree because the person considers himself or herself close to the location of the statement [Agree close (AC)], and (3) Disagree because the person considers himself or herself above the location of the statement [Disagree above (DA)]. As shown in Figure 1, the three responses take the form of responses to three graded response categories. Accordingly, a model for three graded response categories can be applied to this structure.

The model applied is the Rasch (1961) model that can take a number of forms, all equivalent to 
$P\left\{y_{n i}\right\}=\frac{1}{\gamma_{n i}} \exp \left[-\sum_{k=1}^{y} \tau_{k i}+y\left(\beta_{n}-\delta_{i}\right)\right]$,

where

$y_{n i} \in\{0,1,2, \ldots, m\}$ indicates the $m+1$ successive categories beginning with 0 ,

$\tau_{k i}, k=1, \ldots, m$ are $m$ thresholds dividing the continuum into categories; and

$\gamma_{n i}=\sum_{x=0}^{m} \exp \left[-\sum_{k=1}^{y} \tau_{k i}+y\left(\beta_{n}-\delta_{i}\right)\right]$

is the normalizing factor that ensures that the sum of the probabilities is 1.0 (Andersen, 1977; Andrich, 1978a; Wright \& Masters, 1982).

In this paper, and because the focus is on three categories, $m=2$. Further, without loss of generality (Andrich, 1978a), it is assumed that

$\sum_{k=1}^{m} \tau_{k i} \equiv 0$.

The correspondence between the response and the random variable in Equation 1 then is as follows: $y=0$ $\leftrightarrow \mathrm{DB} ; y=1 \leftrightarrow \mathrm{AC} ; y=2 \leftrightarrow \mathrm{DA}$.

If the distance between the two thresholds is parameterized according to $\left(\tau_{2 i}-\tau_{1 i}\right) / 2=\theta_{i}$, then the location parameter $\delta_{i}$ is halfway between the two thresholds, and $\theta_{1}$ represents the distance from $\delta_{i}$ to each of the thresholds (Andrich, 1982). These parameters are also shown in Figure 1. With only three categories it is instructive and efficient to write the probability of each value explicitly in terms of the parameter $\boldsymbol{\theta}_{i}$. Thus, on simplification,

$$
\begin{aligned}
& P\{y=0\}=\frac{1}{\gamma_{n i}}, \\
& P\{y=1\}=\frac{1}{\gamma_{n i}} \exp \left[\theta_{i}+\left(\beta_{n}-\delta_{i}\right)\right],
\end{aligned}
$$

and

$P\{y=2\}=\frac{1}{\gamma_{n i}} \exp 2\left(\beta_{n}-\delta_{i}\right)$.

The thresholds $\tau_{1 i}$ and $\tau_{2 i}$ define the points on either side of the location $\delta_{i}$ of the statement where the probability of each extreme response becomes greater than that of the middle response: at $\beta_{n}=\delta_{i} \pm \theta_{i}$ the probabilities of AC and DB, or AC and DA, are identical. Thus $\theta_{i}$ is very much like a half-unit of measurement, such as a half centimeter about the points marking centimeters on a ruler. For example, if a ruler is marked in centimeters, then any object deemed to be located within half a centimeter on either side of a particular number of say $u$ centimeters, will be declared to be $u$ centimeters long. Likewise, any location of $\beta_{n}$ of person $n$ within $\pm \theta_{i}$ of $\delta_{i}$ will have the highest probability of being located in the middle category, reflected by an Agree response, and therefore at the location of $\delta_{i}$ of the statement. Consistent with this interpretation, Andrich \& Luo (1993) termed $\theta_{i}$ a unit parameter-it characterizes the natural unit of the statement. Furthermore, and consistent with the idea of a unit, the greater the value of $\theta$, the less accurate the discrimination. This region of the continuum, that is here termed a unit, has been termed the latitude of acceptance in social judgment theory (Coombs, 1964; Sherif \& Hovland, 1961); however, in that literature it has been identified as a person parameter rather than a statement parameter. 
The model characterizes the three implied responses of a person to a statement in the unfolding response process; however, there are only two manifest responses and only one manifest Disagree response. That is, $y=0$ and $y=2$ are not distinguished in the data. To make the model correspond to the data, define a new random variable $X_{n i}$ that takes the values $x_{n i}=0$ when $y_{n i}=0$ or $y_{n i}=2$ and $x_{n i}=1$ when $y_{n i}=1$.

Then

$P\left\{x_{n i}=0\right\}=P\left\{y_{n i}=0\right\}+P\left\{y_{n i}=2\right\}$

and

$P\left\{x_{n i}=1\right\}=P\left\{y_{n i}=1\right\}$.

Inserting the explicit expressions of Equations 4-6 into Equations 7-8 (Andrich \& Luo, 1993) gives

$$
P\left\{x_{n i}=0\right\}=\frac{2 \cosh \left(\beta_{n}-\delta_{i}\right)}{\exp \theta_{i}+2 \cosh \left(\beta_{n}-\delta_{i}\right)}
$$

and

$$
P\left\{x_{n i}=1\right\}=\frac{\exp \theta_{i}}{\exp \theta_{i}+2 \cosh \left(\beta_{n}-\delta_{i}\right)},
$$

which can be written as the single expression

$$
P\left\{x_{n i}\right\}=\frac{1}{\gamma_{n i}}\left(\exp \theta_{i}\right)^{x_{n i}}\left[2 \cosh ^{1-x_{n t}}\left(\beta_{n}-\delta_{i}\right)\right],
$$

where

$$
\gamma_{n i}=\exp \theta_{i}+2 \cosh \left(\beta_{n}-\delta_{i}\right)
$$

is now the normalizing factor.

Equation 11 is the HCMDR. Figure 2 shows two pairs of statements with the same location but different unit values.

In the case that all statements of a set are hypothesized to have the same value of $\theta_{i}$, say $\theta_{i}=\theta$ for all $i$, then Equation 11 specializes to

\section{Figure 2}

Pairs of Statements With the Same Location and Different Unit Parameters and Same Unit and Different Location Parameters

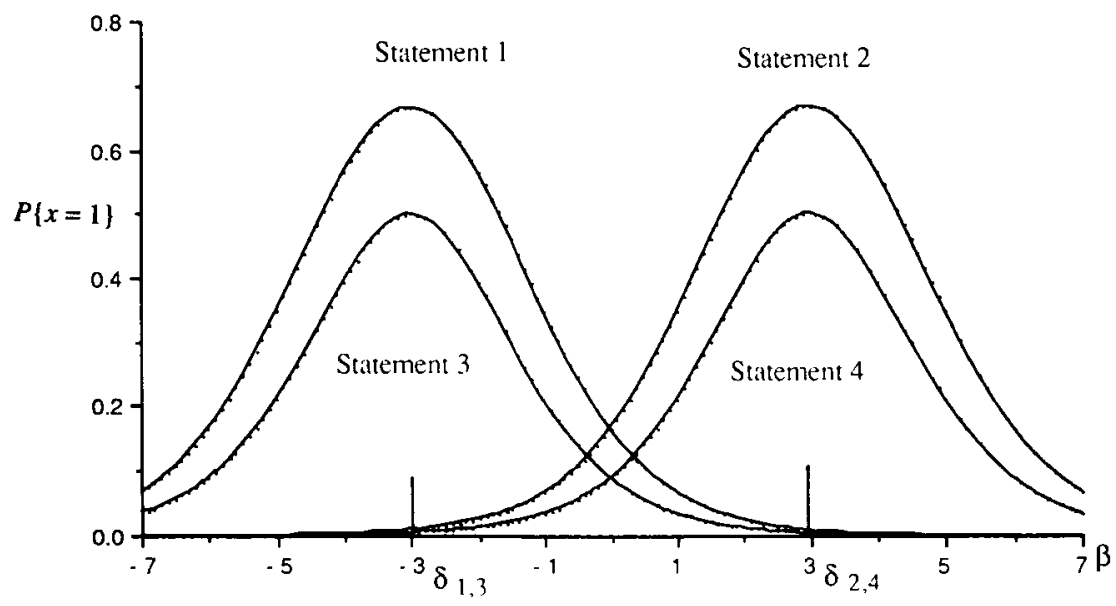


$P\left\{x_{n i}\right\}=\frac{1}{\gamma_{n i}}(\exp \theta)^{x_{n n}}\left[2 \cosh ^{1-x_{n}}\left(\beta_{n}-\delta_{i}\right)\right]$.

Andrich \& Luo (1993) specialized the model of Equation 11 further by setting $\theta=\log 2$ (i.e., $\exp \theta_{i}=2$ ) giving

$P\left\{x_{n i}\right\}=\frac{\cosh ^{1-x_{n i}}\left(\beta_{n}-\delta_{i}\right)}{1+\cosh \left(\beta_{n}-\delta_{i}\right)}$.

This equation has the structure of Rasch's (1960) simple logistic model for the cumulative response process, $P\left\{x_{n i}\right\}=\left[\exp \left(\beta_{n}-\delta_{i}\right)\right]^{x_{n}} /\left[1+\exp /\left(\beta_{n}-\delta_{i}\right)\right]$,

but with the symmetric hyperbolic cosine

$\cosh \left(\beta_{n}-\delta_{i}\right)=\left[\exp \left(\beta_{n}-\delta_{i}\right)+\exp \left(-\beta_{n}+\delta_{i}\right)\right] / 2$

replacing the exponential cumulative function $\exp \left(\beta_{n}-\delta_{t}\right)$. It was this analogy, together with the analogy of $\theta_{i}$ to a unit of measurement, that led Andrich \& Luo (1993) to set $\theta=\log 2$ arbitrarily in Equation 11, which provided Equation 14, and to call Equation 14 a simple hyperbolic cosine model. However, even when $\theta$ is the same for all items, it is not an arbitrary scaling parameter, but a parameter to be estimated, and the estimate is a property of the data (see Appendix A). Thus, the simplest general model for a set of data is one in which all items have the same unit parameter; that is, Equation 13. Therefore, the model of Equation 13 is termed the simple HCMDR (SHCMDR) rather than the model of Equation 14. Thus, the HCMDR (Equation 11) hypothesizes that different items have different estimates of $\theta_{i}$, and the SHCMDR (Equation 13) hypothesizes that $\theta_{i}=\theta$ for all $i$.

It may appear surprising that the unit parameter is not arbitrary, as implied in Equation 14, but rather is a property of the data. This comes from a perspective, portrayed in textbooks in school-level physics, that the unit of measurement may be defined arbitrarily. This arbitrariness, however, is available only up to the choice of unit in making the measurements. Once the unit is selected in an instrument in physical measurement, and if measurement is obtained to the level of precision of the unit, then the precision implied by that unit in any measurement is a property of the measurements and cannot be changed arbitrarily after the data are collected. For example, if measurements taken to the nearest meter are re-expressed in kilometers, the precision of measurement would not be changed to the nearest kilometer-the measurements would still be precise to the nearest meter. In addition, even if the measurements are recorded to some specified unit, they may not be as precise as implied by the unit. Thus measurements of temperature recorded to the nearest 1/10 of a degree Fahrenheit may, on closer empirical examination (and for any number of factors associated with the instrument), be correct only to the nearest $2 / 10$ of a degree. This empirical precision is a property of the data. This is analogous to the HCMDR, except that both the location of the statements (which correspond to the markings on a ruler) and the units about these locations need to be estimated from the data.

\section{Parameter Estimation}

Although the HCMDR is constructed from a Rasch model for ordered categories by combining two categories, the distinguishing feature of Rasch models-the existence of sufficient statistics for the person and statement parameters (Andersen, 1977)-is destroyed. Therefore, methods of estimation that involve conditioning on sufficient statistics for the person and statements are not available. Other methods, including joint maximum likelihood (JML), node estimation (in which persons are provisionally classified into a finite number of groups), the EM algorithm, and marginal maximum likelihood procedures are being investigated. JML was successfully applied by Andrich \& Luo (1993) and is the simplest of these procedures. 
The estimation equations using JML are

$$
\begin{aligned}
& \varphi\left(\delta_{i}\right)=\sum_{n}\left(p_{n i}-x_{n i}\right) \tanh \left(\beta_{n}-\delta_{i}\right)=0, \quad i=1, \ldots, I, \\
& \varphi\left(\beta_{n}\right)=\sum_{n}\left(p_{n i}-x_{n i}\right) \tanh \left(\beta_{n}-\delta_{i}\right)=0, \quad n=1, \ldots, N, \\
& \varphi\left(\theta_{i}\right)=\sum_{n}\left(p_{n i}-x_{n i}\right)=0, \quad i=1, \ldots, I,
\end{aligned}
$$

and

$\sum_{i}\left(\hat{\delta}_{i}\right)=0$,

where $p_{n i}=P\left\{x_{n i}=1\right\}$.

Andrich \& Luo (1993) presented a solution algorithm, initial estimates, and simulation studies that demonstrated the viability of the application of the model. The initial estimates and the interpretation of the estimates must take into account that they are not unique, because two locations give the same probability of a positive response.

In Andrich \& Luo (1993), estimates of the unit parameter $\theta_{i}$ were made, but the mean of these was constrained to $\log 2$. In the algorithm presented here, a single parameter $\hat{\theta}$ is estimated for all of the items first, and then the estimates $\hat{\boldsymbol{\theta}}_{i}$ for each item are obtained with their mean constrained to the common estimate $\hat{\boldsymbol{\theta}}$. Andrich and Luo also noted that for a fixed number of statements and an increase in the number of persons, the estimates of the statement parameters would not tend to their actual values. That is, because of the incidental person parameters, the estimates would be inconsistent. This effect appears in all IRT models using JML estimation, including the simple Rasch model for dichotomous responses (Andersen, 1973). For a fixed set of items, however, the effect of the inconsistency is to spread the estimates further than their actual spread, which effectively changes the scale.

\section{Tests of Fit}

In considering tests of fit between data and a model, the perspective taken here was that the model is based on a substantive theory and that the data collection is governed by the model. Therefore, the degree to which the data accord with the model is the degree to which the theory and its operationalization in the data collection are confirmed, and vice versa. In the application of IRT models, the model selected is expected to summarize the responses with respect to some substantive variable in which the items reflect differences in degree on the latent continuum. It can be argued that in the construction of the items, the goal should be to construct items at different locations on the continuum, and that the relative order of the items, which becomes an hypothesis when the data are collected and analyzed, should be made explicit. This theoretical ordering, taken as an hypothesis, is particularly important when the model, which reflects the response process, is single-peaked because, as already indicated, there are always two person locations that give the same probability of a positive response.

A second point regarding tests of fit is that no test of fit for the HCMDR (or for most models) is necessary and sufficient, and that different tests can be constructed that test different possible violations between the data and the model with different degrees of power. Thus, the question of whether the data fit the model (and by implication the substantive theory) is considered a relatively meaningless question. The questions that were considered more meaningful are the degree to which the data confirm the model and the theory and how the data confirm the model and the theory. In summary, the model that reflects the substantive theory is taken as an hypothesis about the data. If the substantive theory is strong and if the data do not fit the model, then they need to be examined before the theory, and the model that reflects it, are abandoned. 
This perspective is consistent with that articulated by Kuhn (1961, p. 193) regarding physical measurement.

The hypothesis that the unit $\theta_{1}$ is equal for all items can be tested by the usual likelihood ratio $\chi^{2}$ test:

$\chi^{2}=-2 \log \left(L_{\hat{\theta}} / L_{\hat{\theta}_{1}}\right)$

with $I-2$ degrees of freedom $(d f)$ where $L_{\theta}$ is the likelihood under the assumption of $\theta_{i}=\theta$, and $L_{\theta_{i}}$ is the likelihood when each $\theta_{i}$ is estimated.

The second general hypothesis is that the responses take a single-peaked form. This can be evaluated by estimating the locations of the persons and the statements, dividing the persons into class intervals, and determining if the proportions of persons responding positively across class intervals take a single-peaked form. This is a common general test of fit, and can be formalized as a Pearson $\chi^{2}$ statistic

$\chi^{2}=\sum_{i=1}^{I} \sum_{g=1}^{G} \frac{\left[\sum_{n e g} x_{n i}-\sum_{n e g} \mathrm{E}\left(x_{n i}\right)\right]^{2}}{\sum_{n e g} V\left[X_{n i}\right]}=\sum_{i=1}^{I} \sum_{g=1}^{G} \frac{\left[\sum_{n e g} x_{n i}-\sum_{n e g} p_{n i}\right]^{2}}{\sum_{n e g} p_{n i}\left(1-p_{n i}\right)}$,

where $g=1, \ldots, G$ are the class intervals. Asymptotically, as the number of persons and items increases, this statistic should approximate the $\chi^{2}$ distribution with $(G-1)(I-1) d f$. Technically, persons should be classified into class intervals first and then a single location parameter should be estimated for the class interval, rather than a parameter estimated for each person and then probabilities summed. However, in order to be able to order them and place them into class intervals, it is necessary to estimate the person parameters first. Therefore, Equation 22 is used as a guide to the test of general fit. However, the $\chi^{2}$ distribution should only be used as a frame of reference, because sample sizes may not be large enough to satisfy the asymptotic properties or they may be too large, which makes the test of fit unduly sensitive to departures from the model. The power of this test of fit is also governed by the relative locations of the persons and the statements. The greater the variation among locations of statements and persons, the greater the power. If all statements and all persons are located at one position, then there would be no power because there would be no defined continuum, only a point.

However, a simpler and yet complementary procedure is to display the responses of the persons to the statements when both are ordered according to their estimated locations. Then the empirical order of the statements should agree with the theoretical ordering, which in the case of attitude statements is according to their hypothesized affective values. The matrix of responses would then show the parallelogram or diagonal form of Table 1 .

\section{An Example Application}

This example involves the measurement of attitudes toward capital punishment using eight statements originally constructed by Thurstone's methods and subsequently studied again by Wohlwill (1963) and Andrich (1988). 41 people from an educational measurement class at Murdoch University, Australia (Andrich, in press) responded to these items.

Table 2 shows the content of the eight statements. Table 3 shows their estimated affective values ( $\hat{\delta}$ and $\hat{\theta})$ and standard errors ( $\hat{\sigma})$ under the SHCMDR. The statements were assumed to have the same unit $\theta$ for one analysis (Equal Unit $\theta$ ); for the second analysis the unit $\theta_{i}$ was free to vary among statements (Estimated $\theta_{1}$ ). Table 4 shows the frequencies of all observed patterns, the $\hat{\beta}$, and their standard errors $(\hat{\sigma})$.

The sample size for this example may be considered small. However, the advantage of a smaller sample size is that the analysis can be studied very closely, and perhaps more importantly, it shows that the model can 
Table 2

Statements About Capital Punishment

\begin{tabular}{l}
\hline Statement \\
1. Capital punishment is one of the most hideous practices of our time. \\
2. The state cannot teach the sacredness of human life by destroying it. \\
3. Capital punishment is not an effective deterrent to crime. \\
4. I don't believe in capital punishment but I am not sure it isn't necessary. \\
5. I think capital punishment is necessary but I wish it were not. \\
6. Until we find a more civilized way to prevent crime we must have capital punishment. \\
7. Capital punishment is justified because it does act as a deterrent to crime. \\
8. Capital punishment gives the criminal what he deserves.
\end{tabular}

be applied successfully even in the case of small samples. In this case, there is already strong theoretical and empirical evidence that the statements work as a scale; thus, the analysis takes a confirmatory role.

In Table 3 the statements are ordered according to their estimated affective values; in Table 4 the persons are ordered according to the estimates of their attitudes. This helps clarify the definition of the variable and is useful in evaluating fit.

Table 3

Scale Values of Statements About Capital Punishment From Direct Responses for the SHCMDR and HCMDR

\begin{tabular}{|c|c|c|c|c|c|c|c|c|}
\hline \multirow[b]{2}{*}{ Statement } & \multicolumn{4}{|c|}{ Equal Unit $\theta$} & \multicolumn{4}{|c|}{ Estimated $\theta_{i}$} \\
\hline & $\hat{\delta}_{i}$ & $\hat{\sigma}\left(\delta_{t}\right)$ & $\hat{\theta}$ & $\hat{\sigma}(\theta)$ & $\hat{\delta}_{i}$ & $\hat{\sigma}\left(\delta_{i}\right)$ & $\hat{\theta}$ & $\hat{\sigma}(\theta)$ \\
\hline 1 & -10.11 & 1.14 & 6.38 & .23 & -9.80 & 1.10 & 6.54 & 1.09 \\
\hline 2 & -7.78 & 1.00 & 6.38 & .23 & -7.83 & .93 & 6.75 & .93 \\
\hline 3 & -4.24 & .50 & 6.38 & .23 & -5.11 & .52 & 7.69 & .52 \\
\hline 4 & -2.45 & .47 & 6.38 & .23 & -2.26 & .48 & 5.78 & .98 \\
\hline 5 & 3.20 & .79 & 6.38 & .23 & 3.29 & .71 & 5.21 & .71 \\
\hline 6 & 6.06 & .82 & 6.38 & .23 & 6.44 & .82 & 6.60 & .82 \\
\hline 7 & 6.65 & .75 & 6.38 & .23 & 6.79 & .73 & 6.34 & .73 \\
\hline 8 & 8.67 & .58 & 6.38 & .23 & 8.47 & .56 & 6.09 & .56 \\
\hline
\end{tabular}

The ordering of the statements in Table 3 shows that they start at an attitude strongly against capital punishment, move to an ambivalent attitude, and then to an attitude that is strongly for capital punishment. This ordering is also consistent with that found by Wohlwill (1963) and Andrich (1988). In addition, $\mathrm{H}_{0}: \boldsymbol{\theta}_{i}$ $=\theta$ (Equation 21$)$ is tenable $\left(\chi^{2}=6.64, d f=7, p>.36\right)$. This was further confirmed by the closeness of the estimates of the affective values of statements when equal and unequal units were assumed (see Table 3 ) and likewise for the estimates of the attitudes of the persons (see Table 4). The ordering of the persons in Table 4 also shows the required feature, displayed in Table 1, of 1s in a parallelogram around the diagonal for the response patterns.

The likelihood ratio $\chi^{2}$ test of fit of Equation 21 only reflects whether the unit parameter $\theta_{i}$ can be assumed to be homogeneous across items; it does not indicate whether the data in general fit the model. The global test of fit according to Equation 22, in which the sample was divided into three class intervals and the single value of $\hat{\theta}$ was estimated for all items, had a $\chi^{2}=18.00, d f=14, p>.21$, which confirms that the data fit the model. Note that the value of the common unit parameter was estimated to be 6.38 , and not $\log 2=.69$, which was the arbitrary value specified by Andrich \& Luo (1993). Constraining the unit parameter to a value of $\log 2$ increased the $\chi^{2}$ test of fit of Equation 22 from $\chi^{2}=18.00$ to $\chi^{2}=122.99, d f=14, p<<.001$. This result, along with the large difference between $\theta=.69$ and $\hat{\theta}=6.38$ (approximate standard error of .23) makes it unnecessary to conduct a likelihood ratio $\chi^{2}$ test to evaluate specifically whether $\hat{\theta}=6.38$ is significantly different 
Table 4

Distribution of Attitude Scale Values

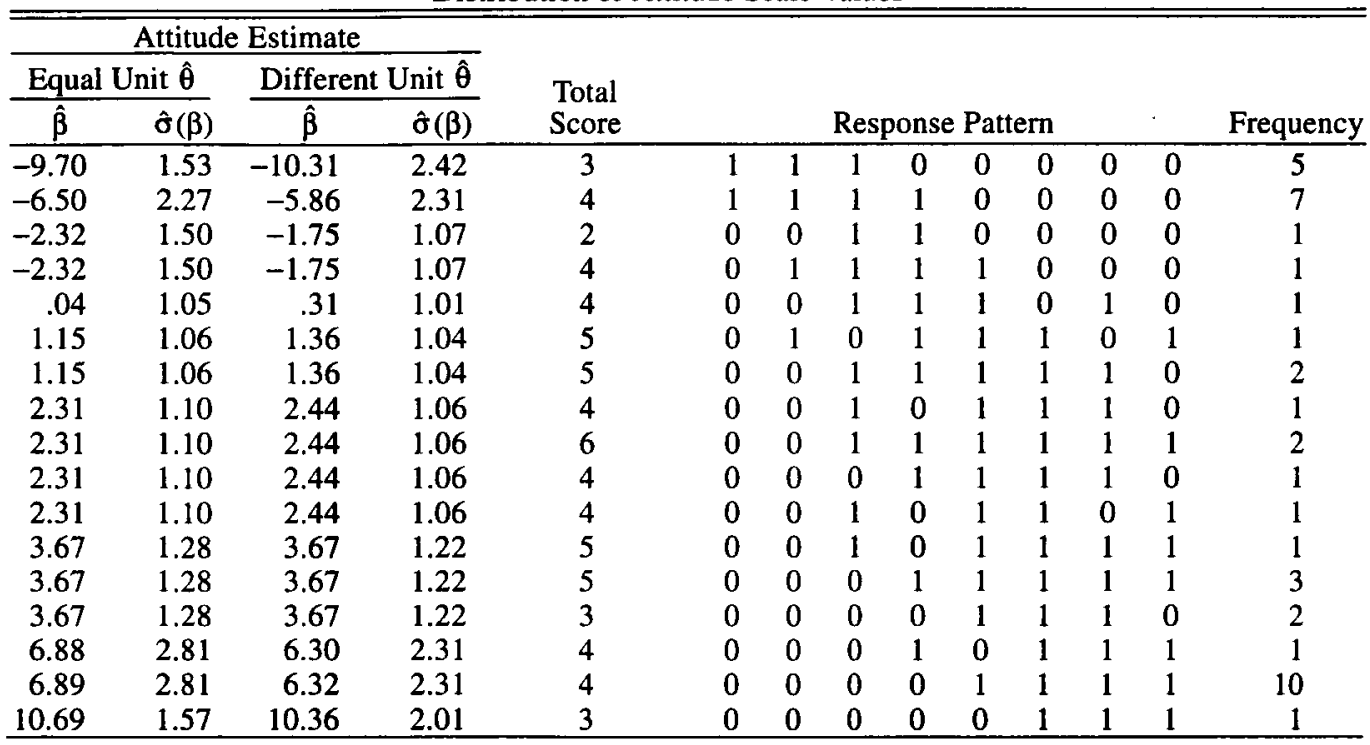

from $\log 2=.69$. This also confirms empirically that the unit parameter is not arbitrary.

Figure 3 shows the locations of the statements and the distribution of persons. The person distribution in Figure 3 is bimodal. Given the evidence that the data conform to the SHCMDR (i.e., the HCMDR with equal units), Figure 3 summarizes the effort to locate statements and persons simultaneously on an attitude continuum. Before proceeding, note that four other people were dropped from the dataset because their individual profiles were very inconsistent with the theoretical ordering of the responses. However, this editing then was followed through into the analysis of the second dataset (described below), and so was not simply a

Figure 3

Locations of Statements and Distribution of Locations of Persons Obtained From Direct Responses and the HCMDR

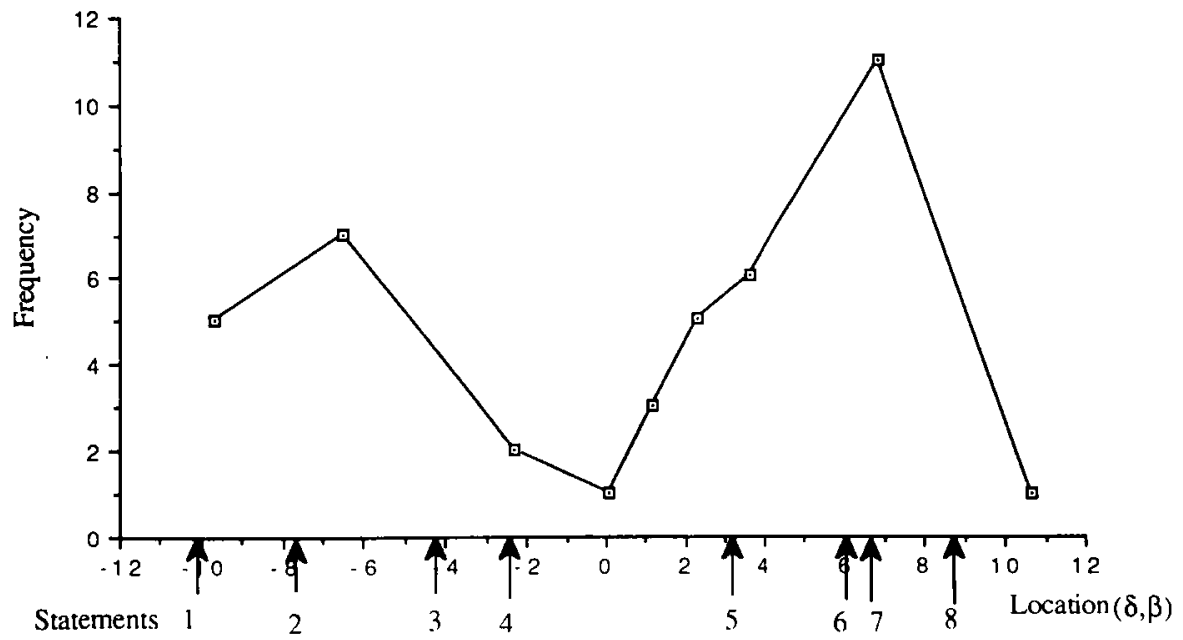


post hoc editing. Indeed, the relationship between the two examples, and the validity of the editing, is important in illustrating aspects of testing model fit and constructing a valid scale with the unfolding process.

\section{The Hyperbolic Cosine Model for Pairwise Preferences}

To emphasize the distinction between pair-comparisons when the cumulative and unfolding response processes are involved, in the latter case they are often termed pairwise preferences. This terminology makes it clear that persons select the statement in each pair that is closest to their ideal point.

The derivation of the model for pairwise preferences from the HCMDR is based on the argument used in Andrich (1978b, 1989). Andrich (1978b) demonstrated how the LCJ (involving, therefore, the cumulative response process) could be derived in an identical form using either a continuous discriminal process of each person to each statement as conceived by Thurstone, or a discrete response process of each person to each statement as in IRT. Andrich (1989) demonstrated how the same unfolding pairwise preference model based on squared distances between the location of the persons and statements could be derived using either a continuous discriminal process (analogous to that used by Thurstone) or a discrete response process of each person to each statement (analogous to that in IRT). Given these identical results using either continuous or discrete response processes, the derivation below is based on the discrete response process of each person to each statement according to the HCMDR presented here.

\section{A Conditional Probability Construction of Pairwise Preferences}

If a person is to select one of two statements, the person must encounter and implicitly react to both statements. If the person makes an implicit discrete dichotomous response to each statement, then there are four possible responses. Assuming stochastic independence and the HCMDR, their probabilities are shown in Table 5. If the person's implicit response is either $(0,0)$ (Disagree to both statements) or $(1,1)$ (Agree to both statements), then there is no basis from which to select one statement over the other. If the response is $(1,0)$ (Agree to the first and Disagree to the second), then the person will select the first statement of the pair; if the response is $(0,1)$ (Disagree to the first and Agree to the second), then the person will select the second statement of the pair. If person $n$ is disposed initially to respond either $(0,0)$ or $(1,1)$ but is required to select between the two statements, suppose that the person reconsiders the response and that the distri-

Table 5

Probabilities of Outcomes for Two Statements $i$ and $j$ According to the HCM,

Where $\gamma_{n i}=\exp \theta_{i}+2 \cosh \left(\beta_{n}-\delta_{i}\right)$ and $\gamma_{n j}=\exp \theta_{j}+2 \cosh \left(\beta_{n}-\delta_{j}\right)$

\begin{tabular}{lll}
\hline \hline$i$ & $j$ & Probability \\
\hline 0 & 0 & $\frac{2 \cosh \left(\beta_{n}-\delta_{i}\right) 2 \cosh \left(\beta_{n}-\delta_{j}\right)}{\gamma_{n i} \gamma_{n j}}$ \\
1 & 0 & $\frac{\exp \theta_{i} 2 \cosh \left(\beta_{n}-\delta_{j}\right)}{\gamma_{n i} \gamma_{n j}}$ \\
0 & 1 & $\frac{2 \cosh \left(\beta_{n}-\delta_{i}\right) \exp \theta_{j}}{\gamma_{n i} \gamma_{n j}}$ \\
1 & 1 & $\frac{\exp \theta_{i} \exp \theta_{j}}{\gamma_{n i} \gamma_{n j}}$ \\
\hline
\end{tabular}


bution of these initial responses is such that the responses $(1,0)$ and $(0,1)$ (from which a preference emerges immediately) retain their original relative probabilities.

The probability of the outcome $(1,0)$, which implies the choice of statement $i$ over $j$, relative to the probability of the outcome $(1,0)$ or $(0,1)$, which implies a choice of one of the statements over the other, is given by

$P_{n i}=\frac{P\{1,0\}}{P\{(1,0) \text { or }(0,1)\}}$.

By substituting Equations 9 and 10 and simplifying, this conditional distribution is given by

$$
P_{n i j}=\frac{\exp \theta_{i} \cosh \left(\beta_{n}-\delta_{j}\right)}{\exp \theta_{i} \cosh \left(\beta_{n}-\delta_{j}\right)+\exp \theta_{j} \cosh \left(\beta_{n}-\delta_{i}\right)} .
$$

Equation 24 is the general hyperbolic cosine model for pairwise preferences (HCMPP). If, by analogy to Thurstone's Case V of the LCJ, the unit $\theta_{\imath}$ is hypothesized to be identical across statements, then Equation 24 reduces to

$$
P_{n j}=\frac{\cosh \left(\beta_{n}-\delta_{j}\right)}{\cosh \left(\beta_{n}-\delta_{j}\right)+\cosh \left(\beta_{n}-\delta_{i}\right)} \text {. }
$$

Equation 25 is referred to as the simple HCMPP (SHCMPP), and is the model further developed and applied here. Models for pairwise preferences generally and implicitly assume an equal unit for the items; however, whether that is the case with any dataset is an empirical question. To summarize the distinction between this model and the LCJ, which is based on the cumulative response process, Figure 4 shows the probability of the endorsement of $i$ over $j$ as a function of $\delta_{i}$ for a fixed $\delta$, and $\beta_{n}$. For the LCJ, this probability is independent of $\beta_{n}$ and increases as $\delta_{i}$ increases relative to $\delta_{j}$. For the SHCMPP it reaches its peak when $\delta_{i}=\beta_{n}$.

Figure 4

RFs for the Cumulative and Unfolding Models for the Endorsement of $i$ Over $j$ as a Function of the Location of $i$ for a Fixed Location of $j$ and Fixed Location of the Person

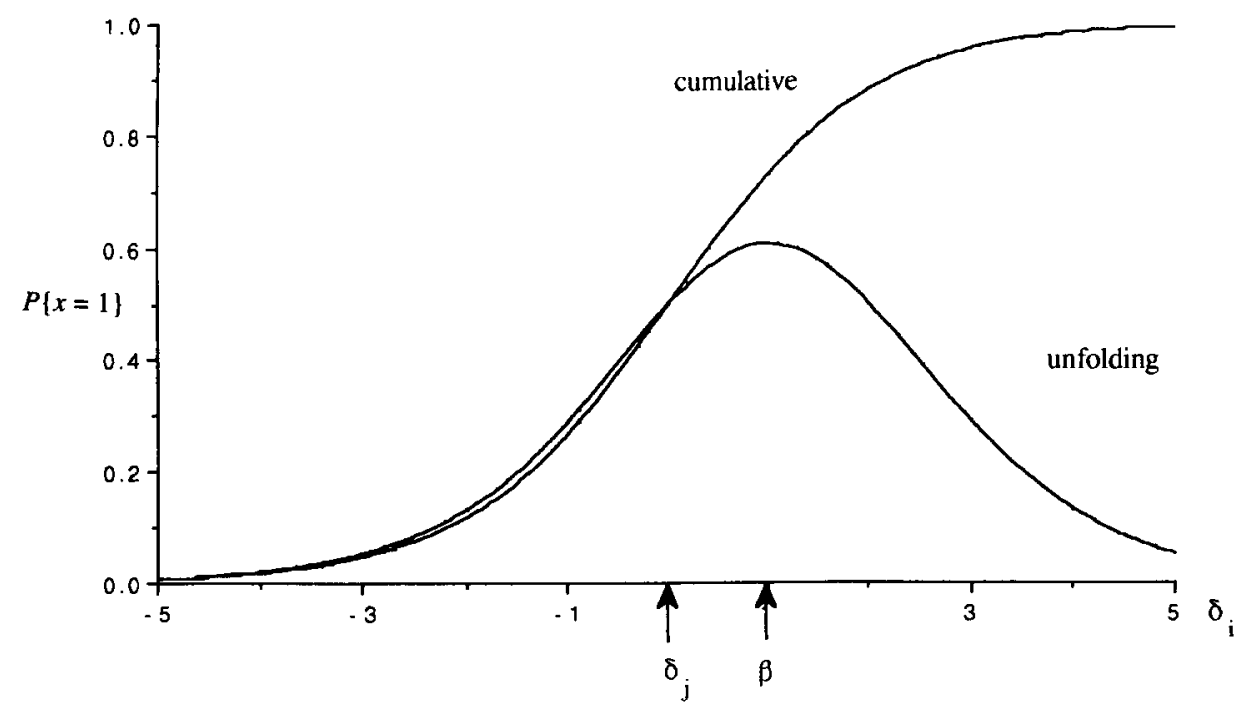


The SHCMPP has a number of important properties. First, in symmetric models for pairwise preferences, $p_{n i j}$ is expected to be exactly .5 when either $\theta_{n}=\left(\delta_{i}+\delta_{j}\right) / 2$ (i.e., when the person is located exactly at the midpoint between the location of the two statements) or when $\delta_{i}=\delta_{j}$ (i.e., when the items have identical locations) irrespective of the location of the person (see Appendix B).

Second, when a person is located on one side of three statements (a situation that also exists if all persons are located on one side of more than three statements), then the relationship between the person and the statements is said to be unilateral. Alternatively, if the person is between one pair of the three statements, then the relationship is said to be bilateral. Transitivity relationships are expected to hold across three statements; these can take various forms, more or less strong. Bossuyt (1990) summarized these possibilities in his comprehensive comparison of the various models for pairwise preferences. Strong stochastic transitivity is said to occur if

$$
p_{n j j} \geq \frac{1}{2} \text { and } p_{n j k} \geq \frac{1}{2} \rightarrow p_{n i k}>\max \left\{p_{n i j}, p_{n j k}\right\} \text {, }
$$

for all statements $i, j$, and $k$. The SHCMPP satisfies strong stochastic transitivity (see Appendix B).

Third, another relationship can be shown to exist between the SHCMPP and Case V of the LCJ. If the logistic response model replaces the normal response model used by Thurstone, then the LCJ takes the form

$p_{i j}=\exp \left(\delta_{i}-\delta_{j}\right) /\left[1+\exp \left(\delta_{i}-\delta_{j}\right)\right]$,

and is independent of $\beta_{n}$ (Andrich, 1978b). This is also the Bradley \& Terry (1952) and Luce (1959) model for choice data under a cumulative model.

In the SHCMPP, as the location of the person gets further away from the location of the two statements in the direction in which it is closer to $\delta_{i}$ than $\delta_{j}$, the probability converges to Equation 27 ; that is,

$$
\lim _{\beta_{n} \rightarrow \infty} p_{n i j}=\exp \left(\delta_{i}-\delta_{j}\right) /\left[1+\exp \left(\delta_{i}-\delta_{j}\right)\right] \text {. }
$$

Again, the proof is shown in Appendix B. Figure 5 shows the relationship between Equations 27 and 28. This mathematical relationship reflects the well-known qualitative effect that if all persons are unilateral relative to all the statements, then the cumulative and unfolding response processes cannot be distinguished in the data. Therefore, for the SHCMPP and all models for pairwise preferences to be applied, it is necessary for the persons to be distributed across the same range of the continuum as the statements. The distribution need not be normal or uniform but it must be as far from unilateral as possible in order to obtain meaningful parameter estimates.

\section{Estimation}

The JML solution equations are presented here for the SHCMPP. In order to formalize the estimates, introduce the dichotomous random variable $X_{n i j}$ that takes the value $x_{n i j}=1$ if person $n$ selects $i$ over $j$ and 0 otherwise. Then Equation 26 takes the form

$$
P\left\{x_{n i j}\right\}=\frac{\cosh ^{\left(1-x_{n j}\right)}\left(\beta_{n}-\delta_{i}\right) \cosh ^{\left(x_{n j}\right)}\left(\beta_{n}-\delta_{j}\right)}{\eta_{n j}},
$$

where

$$
\eta_{n i j}=\cosh \left(\beta_{n}-\delta_{i}\right)+\cosh \left(\beta_{n}-\delta_{j}\right)
$$

is a normalizing factor. 
Figure 5

Limiting Case of the SHCMPP to the LCJ for the Endorsement of $i$ Over $j$ as the Location of the Person Tends to $\pm \infty$

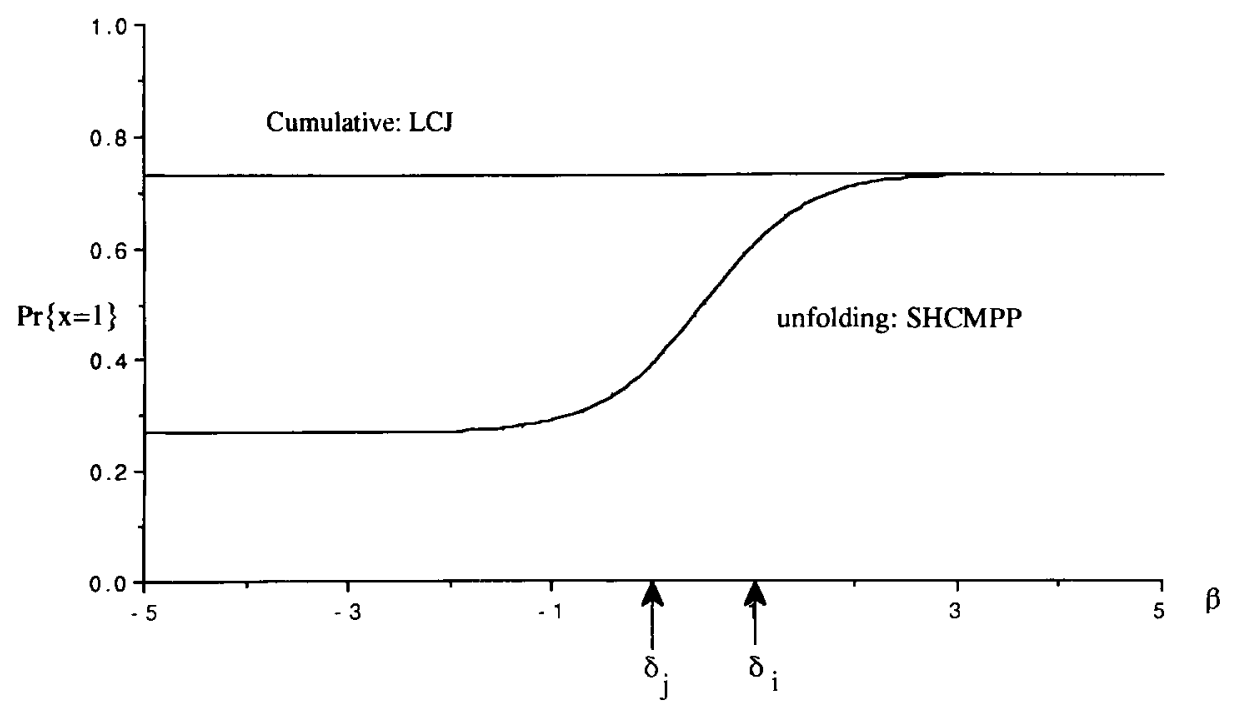

The logarithm of the probability (likelihood of the matrix of observations) then is given by

$\log L=\sum_{n} \sum_{i} \sum_{j}\left[\left(1-x_{n i j}\right) \log \cosh \left(\beta_{n}-\delta_{i}\right)+x_{n i j} \log \cosh \left(\beta_{n}-\delta_{j}\right)-\log \eta_{n i j}\right]$.

Differentiating partially with respect to $\beta_{n}$ gives, on simplification, the solution equation

$\varphi\left(\beta_{n}\right)=\frac{\partial \log L}{\partial \beta_{n}}=\sum_{i=1}^{I} \sum_{j j \neq i}^{I}\left(x_{n i j}-p_{n i j}\right)\left[\tanh \left(\beta_{n}-\delta_{j}\right)-\tanh \left(\beta_{n}-\delta_{i}\right)\right]=0$,

where $n=1, \ldots, N$. Differentiating partially with respect to $\delta$, gives the solution equation

$\varphi\left(\delta_{i}\right)=\frac{\partial \log L}{\partial \delta_{i}}=\sum_{n=1}^{N} \sum_{j, j \neq i}\left(x_{n i j}-p_{n i j}\right) \tan \left(\beta_{n}-\delta_{i}\right)=0$.

One constraint (effectively the origin) is required. This may be set to

$\sum_{i=1}^{1} \hat{\boldsymbol{\delta}}_{i}=0$.

These equations are solved iteratively using the Newton-Raphson algorithm.

Ignoring the diagonal elements of the matrix of second derivatives (as is standard practice in JML estimation; Andrich \& Luo, 1993) gives the following approximate standard errors of the estimates:

$\hat{\sigma}_{\beta_{n}}=-1 / \varphi^{\prime}\left(\beta_{n}\right)=-1 / E\left[\frac{\partial^{2} \log L}{\partial \beta_{n}^{2}}\right]=-1 / \sum_{i} \sum_{i j \neq i} p_{n i j}\left(1-p_{n i j}\right)\left[\tanh \left(\beta_{n}-\delta_{j}\right)-\tanh \left(\beta_{n}-\delta_{i}\right)\right]^{2}$

and

$\hat{\sigma}_{\delta_{i}}=-1 / \varphi^{\prime}\left(\delta_{i}\right)=-1 / E\left[\frac{\partial^{2} \log L}{\partial \delta_{i}^{2}}\right]=-1 / \sum_{n} \sum_{j, j \neq i} p_{n i j}\left(1-p_{n j}\right)\left[\tanh \left(\beta_{n}-\delta_{J}\right)\right]^{2}$. 
The Newton-Raphson algorithm at the $q$ th iteration then takes the form

$\hat{\beta}_{n}^{q+1}=\hat{\beta}_{n}^{q}-\left[\frac{\varphi\left(\beta_{n}\right)}{\varphi^{\prime}\left(\beta_{n}\right)}\right]^{(q)}, \quad n=1, \ldots, N$

and

$\hat{\delta}_{i}^{(q+1)}=\hat{\delta}_{i}^{(q)}-\left[\frac{\varphi\left(\delta_{i}\right)}{\varphi^{\prime}\left(\delta_{i}\right)}\right]^{(q+1)}, \quad i=1, \ldots, I$

for the estimates of $\beta_{n}$ and $\delta_{i}$, respectively.

In setting the initial estimates for the statements, the locations of the persons are assumed not to be unilateral and to be spread more or less evenly among the statements. In that case, statements close to the middle will be selected more often than statements at the extreme.

If

$s_{i}=\sum_{n} \sum_{j, j \neq i} x_{n i}$

is the number of choices that statement $i$ receives and $N$ is the number of persons, then the ratio $\left(N-s_{i}\right) / s_{i}$ has smaller values when $s_{i}$ is large and greater values when $s_{i}$ is small; therefore, it is used as a basis for an initial estimate of $\delta_{i}$. Because the probability of endorsement is a symmetric function of the distances, the hyperbolic cosine is invoked. After some experimentation, and noting that $\cosh (a) \geq 1$, the initial estimate settled on satisfied

$\cosh \left[\delta_{i}^{(0)}\right]=I\left(N-s_{i}\right) / 2 s_{i}$

that is,

$\delta_{i}^{(0)}= \pm \cosh ^{-1}\left[I\left(N-s_{i}\right) / 2 s_{i}\right]$,

where $I$ is the number of statements. The choice of sign begins the unfolding process in which ideally approximately half of the statements should have one sign and the other half the other sign. As in the case of direct responses, this choice of sign requires an a priori theoretical idea of the ordering of the statements or some independent algorithm as described in Andrich \& Luo (1993) for the HCMDR. The solution equations for the SHCMPP do not necessarily guarantee unique or finite estimates. This is not a feature unique to this model, to the solution equations, or to the solution algorithm; rather, it is a function of the singlepeaked nature of the probability function that reflects the data.

Because of the smaller number of items than persons, solutions to person parameters pose potentially more problems than solutions to statement parameters. First, if a person's responses are entirely consistent with a unilateral location (e.g., if four statements are ordered 1, 2, 3, and 4 and the person selects 1 over all statements, 2 over 3 and 4, and 3 over 4), then a finite solution may or may not emerge. In any case, convergence will tend to be slow in the final stages, and the locations and standard errors of the estimates will be large. If there is no convergence, all that is determined is that the person is at an extreme end (i.e., beyond the location of any statement), and how far from the first (or last) statement cannot be determined.

Second, if a person responds very inconsistently in their choices (e.g., if Statements 1, 2, 3, or 4 are ordered and the person selects Statement 1 over all others, and selects Statement 4 over Statements 2 and 3 ), then the likelihood function may have more than one local maximum and again problems with finding a solution might arise. When problems such as these arise in obtaining a solution, they can provide diagnostic information regarding the consistency of a person's responses relative to the theoretical definition 
and operationalization of the variable.

Finally, as in the HCMDR, the estimates will not be consistent, but will spread the items farther than their actual spread. Correction factors and alternative methods of estimation, for both the HCMDR and for the SHCMPP, are being explored but are beyond the scope of this paper.

An appropriate global $\chi^{2}$ test of fit, in which the number of choices $f_{i j}$ for each statement over every other statement is compared to its expected value, can be computed according to

$\chi^{2}=\sum_{i} \sum_{j>i}\left[\left(f_{i j}-\sum_{n} \hat{p}_{n i j}\right)^{2} / \sum_{n} \hat{p}_{n i j}\left(1-\hat{p}_{n i j}\right)\right]$

with $(I-1)(I-2) d f$.

\section{An Example Application}

The number of pairwise choices among $I$ statements is given by $I(I-1) / 2$, and therefore increases rapidly with the number of statements. Therefore, in collecting pairwise preferences from the same sample of persons whose responses were used in the HCMDR example, only Statements 1, 3, 4, 5, and 8 were used. This required the respondents to make 10 pairwise preferences. If all eight statements had been used, 45 comparisons would have been required and would not have been manageable by the respondents in the time available. Each statement was listed first in two comparisons and second in the other two comparisons.

Table 6 shows the estimated scale values of the statements and their standard errors. The observed and expected frequencies for each of the pairwise preferences are in the lower triangle of Table 6; the component $\chi^{2}$ values are in the upper triangle. It is evident that with $\chi^{2}=16.23, d f=6, .01<p<.05$, the data accord reasonably well with the model. One set of preferences-that between Statements 5 and 1 -accounted for 7.91 of the total $\chi^{2}$. The observed and expected frequencies, which were relatively small (thus they inflated the $\chi^{2}$ ), were very similar.

\section{Table 6}

Estimated Scale Values on Pairwise Preferences Among Statements About Capital Punishment (From Table 3): Component $\chi^{2}$ Above the Diagonal, Observed (O) and Expected (E) Frequencies Below the Diagonal

\begin{tabular}{|c|c|c|c|c|c|c|c|c|c|}
\hline \multirow{2}{*}{\multicolumn{2}{|c|}{ Statement }} & \multicolumn{5}{|c|}{ Statement } & \multirow[b]{2}{*}{$\hat{\delta}_{i}$} & \multirow[b]{2}{*}{$\hat{\sigma}\left(\delta_{t}\right)$} & \multirow[b]{2}{*}{$\hat{\delta}_{i}^{a}$} \\
\hline & & 1 & 3 & 4 & 5 & 8 & & & \\
\hline 1 & & & 3.23 & .30 & 7.91 & .18 & -4.00 & .29 & -5.07 \\
\hline 3 & $\begin{array}{l}\mathrm{O} \\
\mathrm{E}\end{array}$ & $\begin{array}{l}23 \\
17.52\end{array}$ & & .02 & .01 & .28 & -2.48 & .22 & -1.81 \\
\hline 4 & $\begin{array}{l}\mathrm{O} \\
\mathrm{E}\end{array}$ & $\begin{array}{l}20 \\
20.72\end{array}$ & $\begin{array}{l}22 \\
21.84\end{array}$ & & 3.40 & .76 & -1.82 & .25 & -.81 \\
\hline 5 & $\begin{array}{l}\mathrm{O} \\
\mathrm{E}\end{array}$ & $\begin{array}{l}18 \\
12.06\end{array}$ & $\begin{array}{l}13 \\
13.28\end{array}$ & $\begin{array}{l}14 \\
16.45\end{array}$ & & .14 & 2.73 & .36 & 2.32 \\
\hline 8 & $\begin{array}{l}\mathrm{O} \\
\mathrm{E}\end{array}$ & $\begin{array}{l}25 \\
25.60 \\
\end{array}$ & $\begin{array}{l}26 \\
26.68 \\
\end{array}$ & $\begin{array}{l}31 \\
29.46 \\
\end{array}$ & $\begin{array}{l}28 \\
28.32 \\
\end{array}$ & & 5.57 & .35 & 5.37 \\
\hline
\end{tabular}

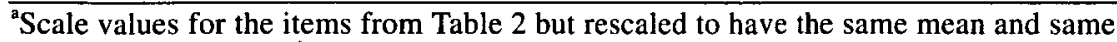
standard deviation as $\hat{\delta}$.

To facilitate comparison of the estimates from the direct-response data and the pairwise preference data, the former were rescaled to a sum of

$\sum_{i=1}^{5} \hat{\delta}_{i}=0$ 
which is the sum of the estimates of the same five statements in the latter. Recall that the sum of the entire set of eight statements from the direct responses was constrained to

$\sum_{i=1}^{8} \hat{\boldsymbol{\delta}}_{i}=0$.

In addition, to account for the possible effect of differences of scale in the two sets of estimates (in part due to different effects of inconsistency) the standard deviation of the estimates from the direct responses was equated to the standard deviation from pairwise preferences.

The scale values were quite similar between those obtained from direct-response data and the SHCMDR. Their correlation was .98 .

Table 7 shows the ordered estimated attitude values and their standard errors as well as the response patterns of the persons. As expected, responses at the extremes provided relatively large estimated values and large standard errors. Because these estimates were from the same persons who provided the direct responses in the HCMDR example, the two sets of estimates were correlated. The product-moment correlation was .84, which indicates that the two methods of data collection (direct response and pairwise preference) when analyzed by complementary models provided similar results. Figure 6 summarizes the location of the statements and the persons for the pairwise preference data.

Table 7

Attitude Estimates, Their Standard Errors, and the Corresponding Response Patterns From Pairwise Preferences (A " 1 " Indicates That the First of the Pair was Chosen, a "0" That the Second of the Pair was Chosen)

\begin{tabular}{|c|c|c|c|c|c|c|c|c|c|c|c|c|}
\hline \multicolumn{10}{|c|}{ Statements in Pairs } & \multirow[b]{2}{*}{$\hat{\beta}$} & \multirow[b]{2}{*}{$\hat{\sigma}_{\beta}$} & \multirow[b]{2}{*}{ Frequency } \\
\hline 31 & 41 & 43 & 51 & 53 & 54 & 81 & 83 & 84 & 85 & & & \\
\hline 0 & 0 & $\mathbf{0}$ & 0 & 0 & 0 & 0 & 0 & 0 & 0 & -6.48 & 150.32 & 6 \\
\hline 0 & 0 & 1 & 0 & 0 & 0 & 0 & 0 & 0 & 0 & -5.64 & 25.32 & 1 \\
\hline 1 & 0 & 0 & 0 & 0 & 0 & 0 & 0 & 0 & 0 & -3.27 & 1.02 & 3 \\
\hline 1 & 0 & 0 & 0 & 0 & 0 & 0 & 0 & 0 & 1 & -3.27 & 1.02 & 1 \\
\hline 1 & 1 & 0 & 0 & 0 & 0 & 0 & 0 & 0 & 0 & -2.15 & 1.22 & 1 \\
\hline 1 & 1 & 0 & 1 & 1 & 0 & 0 & 0 & 0 & 0 & .15 & .54 & 1 \\
\hline 1 & 1 & 1 & 1 & 0 & 0 & 1 & 0 & 0 & 0 & .17 & .54 & 2 \\
\hline 0 & 1 & 1 & 1 & 1 & 1 & 0 & 0 & 0 & 0 & .69 & .52 & 1 \\
\hline 1 & 1 & 1 & 1 & 0 & 1 & 1 & 0 & 0 & 0 & .70 & .52 & 1 \\
\hline 1 & 1 & 1 & 1 & 1 & 1 & 1 & 0 & 0 & 0 & 1.24 & .54 & 2 \\
\hline 1 & 1 & 1 & 1 & 1 & 0 & 1 & 1 & 0 & 0 & 1.27 & .55 & 1 \\
\hline 1 & 1 & 1 & 1 & 1 & 1 & 1 & 1 & 0 & 0 & 1.89 & .63 & 1 \\
\hline 0 & 0 & 0 & 1 & 1 & 1 & 1 & 1 & 1 & 0 & 3.19 & 1.19 & 2 \\
\hline 1 & 0 & 0 & 1 & 1 & 1 & 1 & 1 & 1 & 0 & 3.19 & 1.19 & 1 \\
\hline 1 & 0 & 1 & 1 & 1 & 1 & 1 & 1 & 1 & 0 & 3.19 & 1.19 & 5 \\
\hline 1 & 1 & 1 & 1 & 1 & 1 & 1 & 1 & 1 & 0 & 3.19 & 1.19 & 3 \\
\hline 0 & 0 & 1 & 0 & 1 & 1 & 1 & 1 & 1 & 1 & 7.70 & 145.91 & 1 \\
\hline 0 & 0 & 0 & 1 & 1 & 1 & 1 & 1 & 1 & 1 & 7.90 & 243.12 & 2 \\
\hline 1 & 0 & 0 & 1 & 1 & 1 & 1 & 1 & 1 & 1 & 8.51 & 770.64 & 1 \\
\hline 1 & 1 & 1 & 1 & 1 & 1 & 1 & 1 & 1 & 1 & 8.59 & 912.08 & 5 \\
\hline
\end{tabular}

In interpreting the first example analyzed according to the HCMDR, it was noted that four people whose responses showed gross violations of the order of the statements were eliminated from the dataset. It was surmised that these four persons may have had trouble understanding these statements or were otherwise distracted. If this was the case, then a similar problem should have occurred in their responses to the 
Figure 6

Statement Locations and Frequency Distribution of Persons From Pairwise Preferences and the SHCMPP

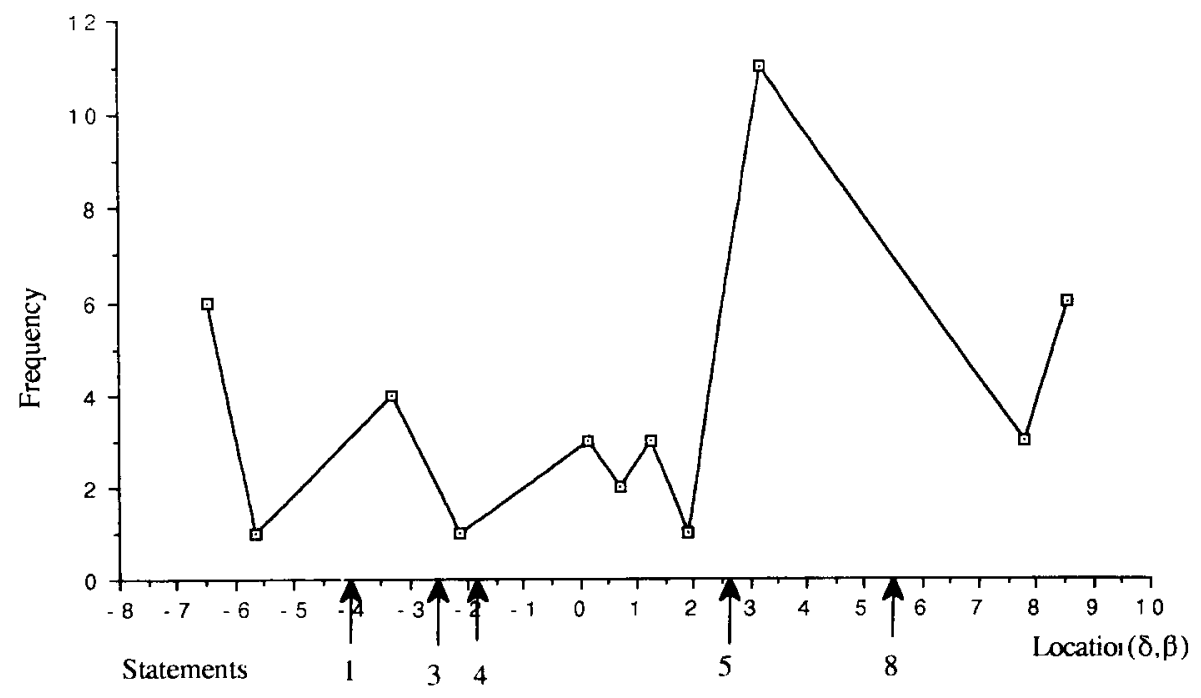

pairwise preferences. Therefore, their responses also were removed from the pairwise preference sample for analysis according to the SHCMPP. With the elimination of these four persons, the general test of fit for the pairwise preferences improved from $\chi^{2}=18.89, d f=6, p<.004$ to $\chi^{2}=16.23, d f=6, .05<p<.01$. This confirmed that the four persons had responses of doubtful validity in both sets of data. Ideally, these persons, if they were known, would be interviewed regarding their responses. Then, either it would be confirmed that they had trouble with the language, or if they did not, that they perhaps interpreted the statements in some different way.

\section{Conclusions}

The data analyzed in the two examples were consistent with the respective models and also were consistent with each other. The scale values in the direct-response design had a greater spread than in the pairwise preference design. This difference in scale was attributed in part to a different effect of the inconsistency of estimates. These effects need to be explored further.

It was also noted that with unfolding models unique solutions are not guaranteed because two different locations give the same probability of any response. Technical problems of this kind can be overcome by having a strong substantive theory for the a priori ordering of the statements.

\section{Appendix A: Demonstration of the Nonarbitrariness of the Unit Parameter $\theta$ in the SHCMDR}

Transformations of parameters, provided they leave the probability and the model unchanged, are permissible and considered to do no more than change an arbitrary metric. Of course, arbitrary metrics may be selected for convenience. Monotonic continuous transformations of real-valued parameters provide such transformations (Rasch, 1960; van der Linden, 1993). For example, consider Rasch's simple logistic model for dichotomous responses that can be expressed in the form

$P_{n i}=P\left\{x_{n i}=1\right\}=\exp \left(\beta_{n}-\delta_{i}\right) /\left[1+\exp \left(\beta_{n}-\delta_{i}\right)\right]$.

A parameter $\alpha$ can be introduced to give the form 


$$
P_{n t}=\exp \left[\alpha\left(\beta_{n}-\delta_{l}\right)\right] /\left\{1+\exp \left[\alpha\left(\beta_{n}-\delta_{i}\right)\right]\right\}
$$

If the items have a different value $\alpha_{i}$ for each item, then the model becomes the two-parameter logistic model (Birnbaum, 1968). However, if $\alpha$ is common to all items, then it provides only an arbitrary scaling of Rasch's (1960) simple logistic model. For example, if

$$
P_{n i}=\frac{\exp \left[\alpha\left(\beta_{n}-\delta_{i}\right)\right]}{1+\exp \left[\alpha\left(\beta_{n}-\delta_{i}\right)\right]}=\frac{\exp \left[\alpha^{*}\left(\beta_{n}^{*}-\delta_{i}^{*}\right)\right]}{1+\exp \left[\alpha^{*}\left(\beta_{n}^{*}-\delta_{i}^{*}\right)\right]},
$$

it can be shown readily that

$$
\beta_{n}^{*}-\delta_{i}^{*}=\frac{\alpha}{\alpha^{*}}\left(\beta_{n}-\delta_{i}\right) \text {. }
$$

Thus, the contrast $\beta_{n}^{*}-\delta_{i}^{*}$ is simply a monotonic (in this case simple linear) scaling of the constrast $\left(\beta_{n}-\right.$ $\delta_{i}$ ). In contrast, a change of value $\theta \rightarrow \theta^{*}$ in the SHCMDR provides a nonmonotonic or discontinuous transformation of $\beta_{n}-\delta_{i} \rightarrow \beta_{n}^{*}-\delta_{i}^{*}$. Thus, if the probability of a positive response $P_{n i}$ and the form of the SHCMDR remains the same when $\theta \rightarrow \theta^{*}$, then

$$
p_{n i}=\frac{\exp \theta}{\exp \theta+2 \cosh \left(\beta_{n}-\delta_{i}\right)}=\frac{\exp \theta^{*}}{\exp \theta^{*}+2 \cosh \left(\beta_{n}^{*}-\delta_{i}^{*}\right)},
$$

from which it can be shown that

$$
\beta_{n}^{*}-\delta_{i}^{*}= \pm \cosh ^{-1}\left[\exp \left(\theta^{*}-\theta\right) \cosh \left(\beta_{n}-\delta_{i}\right)\right] \text {. }
$$

Figure 7 shows this transformation of the contrast $\beta_{n}-\delta_{i}$ to $\beta_{n}^{*}-\delta_{i}^{*}$ for changes of $\theta$ from $\theta=\log 2$ to $\theta^{*}$ $=\log 3, \log 4$, and $\log 5$. If $\beta_{n}^{*}-\delta_{i}^{*}$ is constrained to be positive throughout (shown in the upper part of Figure 7), then the transformation is not monotonic; if $\beta_{n}^{*}-\delta_{i}^{*}$ is assigned the same sign as $\beta_{n}-\delta_{i}$ throughout (the solid lines in Figure 7), then the transformation is not continuous.

\section{Appendix B: Demonstration That in the SHCMPP $p_{n i j}=.5$ When $\beta_{n}=\left(\delta_{i}+\delta_{j}\right) / 2$ or $\delta_{i}=\delta_{j}$}

If $\beta_{n}=\left(\delta_{i}+\delta_{j}\right) / 2$, then

$$
\begin{aligned}
p_{n i j} & =\frac{\cosh \left(\beta_{n}-\delta_{j}\right)}{\cosh \left(\beta_{n}-\delta_{i}\right)+\cosh \left(\beta_{n}-\delta_{J}\right)}=\frac{\cosh \left(\beta_{n}-2 \beta_{n}+\delta_{i}\right)}{\cosh \left(\beta_{n}-\delta_{i}\right)+\cosh \left(\beta_{n}-2 \beta_{n}+\delta_{i}\right)} \\
& =\frac{\cosh \left(-\beta_{n}+\delta_{i}\right)}{\cosh \left(\beta_{n}-\delta_{i}\right)+\cosh \left(-\beta_{n}+\delta_{i}\right)}=\frac{\cosh \left(\beta_{n}-\delta_{i}\right)}{2 \cosh \left(\beta_{n}-\delta_{i}\right)}, \text { because } \cosh (-a)=\cosh (a)=.5 .
\end{aligned}
$$

And if $\delta_{i}=\delta_{j}$, then

$$
p_{n i j}=\frac{\cosh \left(\beta_{n}-\delta_{i}\right)}{2 \cosh \left(\beta_{n}-\delta_{i}\right)}=.5 \text {. }
$$

\section{Demonstration of Stochastic Transitivity in the SHCMPP}

That is, if $p_{n i j} \geq .5$ and $p_{n j k} \geq .5$, then $p_{n i k} \geq \max \left\{p_{n i j}, p_{n j k}\right\}$.

First, it is shown that if $p_{n i j} \geq .5$, then $p_{n i k} \geq p_{n j k}$ for all $k$. (This first part of the proof was provided by G. Luo, personal communication, 1993). 
Figure 7

Transformation of the Latent Continuum as a Function of Three Changes of the Unit Relative to a Standard Unit of Size Log 2 (The Solid Line Assigns the Same Sign to $\beta^{*}-\delta^{*}$ as $\beta-\delta$ )

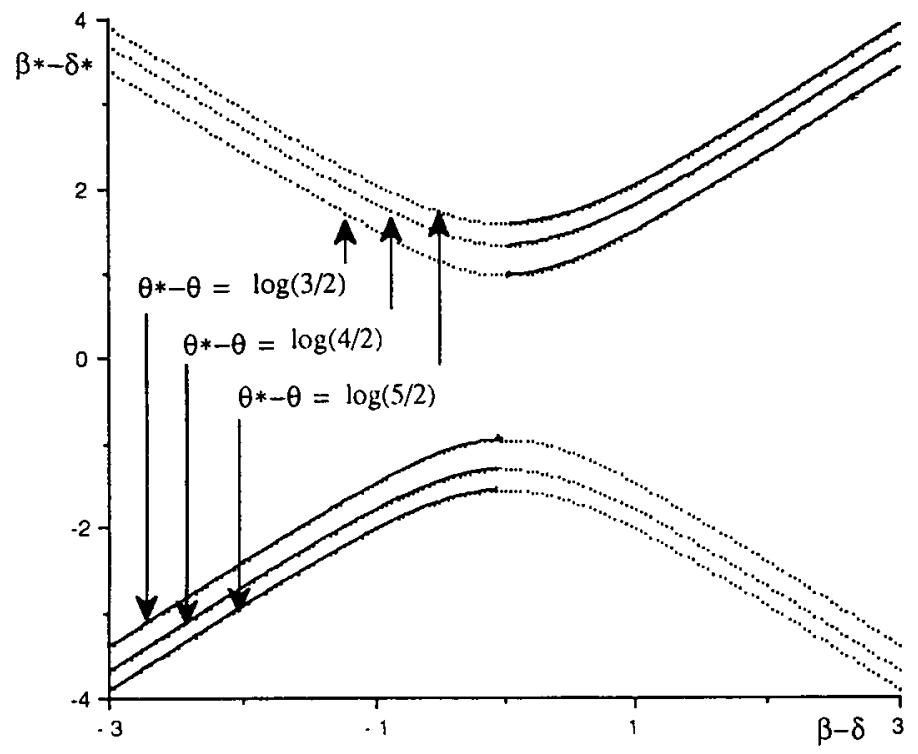

If $p_{n i j} \geq .5$, then

$\frac{\cosh \left(\beta_{n}-\delta_{j}\right)}{\cosh \left(\beta_{n}-\delta_{i}\right)+\cosh \left(\beta_{n}-\delta_{j}\right)} \geq .5$

and

$\frac{\cosh \left(\beta_{n}-\delta_{i}\right)}{\cosh \left(\beta_{n}-\delta_{i}\right)+\cosh \left(\beta_{n}-\delta_{J}\right)} \leq .5$.

Therefore, $\cosh \left(\beta_{n}-\delta_{j}\right) \geq \cosh \left(\beta_{n}-\delta_{i}\right)$.

That is,

$\cosh \left(\beta_{n}-\delta_{\jmath}\right)+\cosh \left(\beta_{n}-\delta_{k}\right) \geq \cosh \left(\beta_{n}-\delta_{i}\right)+\cosh \left(\beta_{n}-\delta_{k}\right)$,

$\frac{1}{\cosh \left(\beta_{n}-\delta_{j}\right)+\cosh \left(\beta_{n}-\delta_{k}\right)} \leq \frac{1}{\cosh \left(\beta_{n}-\delta_{i}\right)+\cosh \left(\beta_{n}-\delta_{k}\right)}$

and

$\frac{\cosh \left(\beta_{n}-\delta_{k}\right)}{\cosh \left(\beta_{n}-\delta_{\jmath}\right)+\cosh \left(\beta_{n}-\delta_{k}\right)} \leq \frac{\cosh \left(\beta_{n}-\delta_{k}\right)}{\cosh \left(\beta_{n}-\delta_{\imath}\right)+\cosh \left(\beta_{n}-\delta_{k}\right)}$.

That is, $p_{n j k} \leq p_{n i k}$ and $p_{n i k}>p_{n j k}$.

Now if $p_{n i k} \geq .5$, then $p_{n i k} \geq p_{n j k}$, 
and if $p_{n j k} \geq .5$, then $p_{n j i} \geq p_{n k i}$,

that is, $p_{n k i} \leq p_{n j i}$ and $p_{n i k} \geq p_{n i j^{\prime}}$

Thus, if $p_{n i j} \geq .5$ and $p_{n j k} \geq .5$,

then $p_{n i k} \geq p_{n j k}$ and $p_{n i k} \geq p_{n i j}$.

Therefore, $p_{n i k} \geq \max \left\{p_{n i j}, p_{n j k}\right\}$.

The Limit of $p_{n j}$ When $\beta \rightarrow \infty$ Relative to Statements $i$ and $j$ and $\theta_{n}$ is Closer to $i$ Than $j$

$p_{n i j}=\frac{\cosh \left(\beta_{n}-\delta_{j}\right)}{\cosh \left(\beta_{n}-\delta_{i}\right)+\cosh \left(\beta_{n}-\delta_{j}\right)}$

and

$$
\begin{aligned}
p_{n i j} & =\lim _{\beta_{n} \rightarrow \infty} \frac{\exp \left(\beta_{n}-\delta_{j}\right)+\exp \left(-\beta_{n}+\delta_{j}\right)}{\exp \left(\beta_{n}-\delta_{i}\right)+\exp \left(-\beta_{n}+\delta_{i}\right)+\exp \left(-\beta_{n}+\delta_{j}\right)+\exp \left(-\beta_{n}+\delta_{j}\right)} \\
& =\lim _{\beta_{n} \rightarrow \infty} \frac{\exp \left(-\delta_{j}\right)+\exp \left(-2 \beta_{n}+\delta_{j}\right)}{\exp \left(-\delta_{i}\right)+\exp \left(-2 \beta_{n}+\delta_{i}\right)+\exp \left(-\delta_{j}\right)+\exp \left(-2 \beta_{n}+\delta_{j}\right)} \\
& =\frac{\exp \left(-\delta_{j}\right)}{\exp \left(-\delta_{i}\right)+\exp \left(-\delta_{j}\right)}=\frac{\exp \left(\delta_{i}-\delta_{j}\right)}{1+\exp \left(\delta_{i}-\delta_{j}\right)} .
\end{aligned}
$$

\section{References}

Andersen, E. B. (1973). Conditional inference for multiple choice questionnaires. British Journal of Mathematical and Statistical Psychology, 26, 31-44.

Andersen, E. B. (1977). Sufficient statistics and latent trait models. Psychometrika, 42, 69-81.

Andrich, D. (1978a). A rating formulation for ordered response categories. Psychometrika, 43, 357-374.

Andrich, D. (1978b). Relationships between the Thurstone and Rasch approaches to item scaling. Applied Psychological Measurement, 2, 451-462.

Andrich, D. (1982). An extension of the Rasch model for ratings providing both location and dispersion parameters. Psychometrika, 47, 105-113.

Andrich, D. (1988). The application of an unfolding model of the PIRT type for the measurement of attitude. Applied Psychological Measurement, 12, 33-51.

Andrich, D. (1989). A probabilistic item response theory model for unfolding preference data. Applied Psychological Measurement, 13, 193-216.

Andrich, D. (in press). Hyperbolic cosine IRT model for unfolding direct responses of persons to items. In W. van der Linden \& R. K. Hambleton (Eds.), Handbook of modern item response theory. New York: Springer-Verlag.

Andrich, D., \& Luo, G. (1993). A hyperbolic cosine latent trait model for unfolding dichotomous single- stimulus responses. Applied Psychological Measurement, 17, 253-276.

Birnbaum, A. (1968). Some latent trait models and their use in inferring an examinee's ability. In F. M. Lord \& M. R. Novick, Statistical theories of mental test scores (pp. 397-479). Reading MA: Addison-Wesley.

Bossuyt, P. (1990). A comparison of probabilistic unfolding theories for paired comparisons data. Berlin: Springer-Verlag.

Bradley, R. A., \& Terry, M. E. (1952). Rank analysis of incomplete block designs 1: The method of paired comparisons. Biometrika, 39, 324-345.

Carroll, J. D. (1972). Individual differences and multidimensional scaling. In R. N. Shepard, A. K. Romney, \& S. Nerlove (Eds.), Multidimensional scaling: Theory and applications in the social sciences (Vol. 1; pp. 105-153). New York: Seminar Press.

Coombs, C. H. (1964). A theory of data. New York: Wiley.

Coombs, C. H., \& Avrunin, C. S. (1977). Single-peaked functions and the theory of preference. Psychological Review, 84, 216-230.

Coombs, C. H., \& Smith, J. E. K. (1973). On the detection of structure in attitudes and developmental process. Psychological Review, 80, 337-351.

Cooper, L. G., \& Nakanishi, M. (1983). Two logit mod- 
els for external preferences. Psychometrika, 48, 607-620.

Davison, M. (1977). On a metric, unidimensional unfolding model for attitudinal and developmental data. Psychometrika, 42, 523-548.

DeSarbo, W. A., \& Hoffman, D. L. (1986). The simple and weighted thresholds model for the spatial representation of binary choice data. Applied Psychological Measurement, 10, 247-264.

Guttman, L. (1950). The basis for scalogram analysis. In S. A. Stouffer (Ed.), Measurement and prediction (pp. 60-90). New York: Wiley.

Kuhn, T. S. (1961/1977). The function of measurement in modern physical science. Isis, 52, 161-193. Reproduced in Kuhn, T. S. (1977). The essential tension (pp. 178-239). Chicago: The University of Chicago Press.

Leik, R. K., \& Matthews, M. (1968). A scale for developmental processes. American Sociological Review, $33,62-75$.

Luce, R. D. (1959). Individual choice behavior. New York: Wiley.

Poole, K. T. (1984). Least squares metric, unidimensional unfolding. Psychometrika, 49, 311-323.

Post, W. J. (1992). Nonparametric unfolding models: A latent structure approach. Leiden: DSWO Press.

Rasch, G. (1960/80). Probabilistic models for some intelligence and attainment tests. (Copenhagen, Danish Institute for Educational Research). Expanded edition (1980), with foreword and afterword by B. D. Wright. Chicago, The University of Chicago Press.

Rasch, G. (1961). On general laws and the meaning of measurement in psychology. In J. Neyman (Ed.), Proceedings of the Fourth Berkeley Symposium on Mathematical Statistics and Probability, IV, 321-334. Berkeley: University of California Press.

Schönemann, P. H. (1970). On metric multidimensional unfolding. Psychometrika, 35, 349-366.

Schönemann, P. H., \& Wang, W. M. (1972). An individual differences model for the multidimensional analysis of preference data. Psychometrika, 37, 275-309.

Sherif, M., \& Hovland, C. (1961). Social judgment: Assimilation and contrast effects in communication and attitude change. New Haven CT: Yale University Press.

Sixtl, F. (1973). Probabilistic unfolding. Psychometrika,
$38,235-248$.

Thurstone, L. L. (1927). A law of comparative judgement. Psychological Review, 34, 278-286.

Thurstone, L. L. (1928). Attitudes can be measured. American Journal of Sociology, 33, 529-54.

van Blokland-Vogelesang, R. (1991). Unfolding and group consensus ranking for individual preferences. Leiden: DSWPO Press.

van der Linden, W. J. (1993). Measurement scale in the Rasch model. Rasch Measurement Transactions, 7, 287.

van Schuur, W. H. (1984). Structure in political beliefs, a new model for stochastic unfolding with application to European party activists. Amsterdam: CT Press.

van Schuur, W. H. (1987). Constraint in European party activists' sympathy scores for interest groups: The left-right dimension as dominant structuring principle. European Journal of Political Research, 15, 347-362.

van Schuur, W. H. (1989). Unfolding German political parties: A description and application of multiple unidimensional unfolding. In G. de Soete, H. Ferger, \& K. C. Klauer (Eds.), New developments in psychological choice modelling (pp. 259-277). Amsterdam: North Holland.

Volet, S. E., \& Chalmers, D. (1992). Investigation of qualitative differences in university students' learning goals, based on an unfolding model of stage development. British Journal of Educational Psychology, 62, 17-34.

Wohlwill, J. F. (1963). The measurement of scalability for non-cumulative items. Educational and Psychological Measurement, 23, 543-55.

Wright, B. D., \& Masters, G. N. (1982). Rating scale analysis: Rasch measurement. Chicago: MESA Press.

\section{Acknowledgments}

This research was funded in part by the Australia Research Council. Graham Douglas and Irene Styles read the paper and made constructive suggestions, as did two unknown reviewers. Guanzhong Luo wrote the programs for the data analysis and provided many useful discus. sions with the author.

\section{Author's Address}

Send requests for reprints or further information to David Andrich, School of Education, Murdoch University, Murdoch, Western Australia 6150. 